\title{
CAPACITIES OF BORELIAN SETS AND THE CONTINUITY OF POTENTIALS
}

\author{
MASANORI KISHI
}

\section{Introduction}

One of the most important problems in the potential theory is the one of capacitability, that is, whether the inner capacity of an arbitrary borelian subset $B$ is equal to the outer capacity of $B$. As for the capacities induced by the Newtonian potentials and other classical potentials, Choquet [5] has shown that every borelian and, more generally, every analytic set are capacitable. He goes on as follows: first he shows that, for the Newtonian capacity $f$, the inequality of strong subadditivity holds, that is,

$$
f(A \cup B)+f(A \cap B) \leqq f(A)+f(B),
$$

and then, using this inequality, he shows that the outer capacity $f^{\prime}$ has the analogous property to one of the outer measure, more precisely, if an increasing sequence $\left\{A_{n}\right\}$ of arbitrary subsets converges to $A$, then $f^{*}(A)$ $=\lim f^{*}\left(A_{n}\right)$. This property plays an important role in his proof.

Recently it tends to investigate the general potentials in a locally compact Hausdorff space. As for the problem of capacitability, anything more than the results of Choquet has not yet been stated. In this paper we deal with this problem and we shall prove that every $K$-borelian subset and, more generally, every $K$-analytic subset, contained in a compact set, are capacitable under the two assumptions that every compact subset is metrisable and there exists en equilibrium measure of every compact subset. $A K$-borelian subset is a subset belonging to the $K$-borelian field, which is the smallest borelian field which contains each compact subset. As every compact set is metrisable in our case, every classical borelian subset contained in a compact set is $K$-borelian. A $K$-analytic subset is the continuous image of a $K_{r \delta}$ set contained in a compact space. It is known that every $K$-borelian subset is $K$-analytic.

First, in $\S 1$, we consider the quasi continuity principle, which is a gener-

Received September 14, 1957. 
alisation of the continuity principle and we shall prove that the quasi continuity principle follows from the assumption that there exists an equilibrium measure of every compact subset. In the following section we shall prove that, if a sequence $\left\{\mu_{n}\right\}$ of positive measures on a compact subset converges vaguely to a measure $\mu$ and if the potentials $U^{\mu_{n}}$ are uniformly bounded, then we have $U^{\mu}=\lim _{n} U^{\mu_{n}}$ quasi everywhere. This fact is very important to assert that the outer capacity, induced by our potentials, has the following property: the sequence of the outer capacities of arbitrary subsets $A_{n}$ in a compact subset converges increasingly to the outer capacity of $A$, whenever $\left\{A_{n}\right\}$ increases to $A$. From this property follows the capacitability of all analytic subsets in a compact set.

In the last section, we associate a function $m^{*}\left(P, U^{\mu}\right)$ with each potential $U^{\mu}$. Using this function, first we shall investigate the continuity of potentials, and then we shall discuss an equilibrium potential of an open set $G$ which is constantly equal to 1 in $G$.

\section{$\S 1$ Quasi continuity principle}

1. Let $\Omega$ be a locally compact Hausdorff space. In the sections 1,2 and 3 we shall assume that every compact subset of $\Omega$ is metrisable. In this paper we always consider positive measures ${ }^{1)} \mu$ in $\Omega$ with compact carriers denoted by $S_{\mu}$. We denote by $\mu(1)$ the total mass of a positive measure $\mu$. $A$ sequence $\left\{\mu_{n}\right\}$ of positive measures is called to converge vaguely to $\mu$, when we have

$$
\int f d \mu=\lim _{n} \int f d \mu_{n}
$$

for every continuous function $f$ with compact carrier.

The following theorem is important in the potential theory.

THEOREM 1.1. ${ }^{2)}$ Suppose that positive measures $\mu_{n}(n=1,2, \ldots)$ satisfy the following conditions:

$1^{\circ} S_{\mu_{n}}(n=1,2, \ldots)$ are contained in a fixed compact subset $K$,

$2^{\circ} \mu_{n}(1)(n=1,2, \ldots)$ are uniformly bounded from above.

1) For the theory of measures in a locally compact Hausdorff space, see, for example, Bourbaki [2].

2) Cf. Frostman [8]. 
Then, from a given $\left\{\mu_{n}\right\}$, we can take out a subsequence $\left\{\mu_{n^{\prime}}\right\}$ which converges vaguely to a positive measure $\mu$.

Since $K$ is metrisable, this theorem can be proved using the following theorem. We shall onit the proof of Theorem 1.1.

TheоRem. ${ }^{3)}$ Suppose that a compact set $K$ is metrisable. Then the space $(S(K)$ of all continuous functions in $K$ with the uniform convergence topology is separable.

Conversely we can prove

THEOREM 1.2. If $(K(K)$ is separable, then the compact set $K$ is metrisable.

Proof. Let $\tilde{F}$ be a countable subset of $\mathfrak{5}(K)$ which is dense in $(5 .(K)$ with respect to the uniform convergence topology. For each point $P_{0}$ of $K$ we define a new base of neighborhoods $N\left(P_{n}\right)$ as follows:

$$
N\left(P_{0}\right)=\left\{P \in K ;\left|f_{j}(P)-f_{j}\left(P_{0}\right)\right|<\varepsilon_{j}, \varepsilon_{j}>0, f_{j} \in \mathfrak{F} ; j=1,2, \ldots, m\right\} .
$$

Let us denote by $\tilde{K}$ the set $K$ with this topology. It is easily seen that $\widetilde{K}$ is a Hausdorff space. In fact, for any two points $P_{1}$ and $P_{2}$ of $K$, there exists a continuous function $f(P)$ of $\left(\xi(K)\right.$ such that $f\left(P_{1}\right)=0$ and $f\left(P_{2}\right)=1$. Then we can choose a function $f_{j_{0}}$ of $\tilde{s}$ such that $\left|f(P)-f_{j_{0}}(P)\right|<\frac{1}{4}$ at every point $P$ of $K$. Put

and

$$
N\left(P_{1}\right)=\left\{P ;\left|f_{j_{0}}(P)-f_{j_{0}}\left(P_{1}\right)\right|<\frac{1}{4}\right\}
$$

$$
N\left(P_{2}\right)=\left\{P ;\left|f_{j_{0}}(P)-f_{j_{0}}\left(P_{2}\right)\right|<\frac{1}{4}\right\}
$$

Then we see that $N\left(P_{1}\right) \cap N\left(P_{2}\right)=\emptyset$. It is easy to verify that $K$ and $\tilde{K}$ are homeomorphic, and that by our topology $\tilde{K}$ satisfies the first axiom of countability, and so does $K$.

Now we shall show that $K$ is separable. For the purpose, first we show that, for any neighborhood $U$ of an arbitrary point $P_{0}$ of $K$, there exists a continuous function $f(P)$ of $\left(\mathrm{S}(K)\right.$ such that $0 \leqq f(P) \leqq 1$ in $K, f\left(P_{0}\right)=1, f(P)=0$ at every point $P$ of $K-U$ and $f(P)<1$ at each point $P \neq P_{0}$. In fact, since $K$ satisfies the first axiom of countability, there exists a sequence $\left\{U_{n}\right\}(n=1$,

3) Cf. Kryloff and Bogoliouboff [12]. 
$2, \ldots$ ) of neighborhoods of $P_{0}$ such that

$$
U \supset U_{n} \supset \bar{U}_{n+1} \supset U_{n+1} \text { and } \bigcap_{n} U_{n}=\left\{P_{0}\right\}
$$

Since $K$ is normal by the compactness of $K$, we can find a continuous function $f_{n}(P)$ of $\$(K)$ for each $n$ such that $f_{n}\left(P_{0}\right)=1, f_{n}(P)=0$ at every point $P$ of $K-U_{n}$ and $0 \leqq f_{n}(P) \leqq 1$ in $K$. Then we see that the continuous function $f(P)=\sum \frac{1}{2^{n}} f_{n}(P)$ satisfies our requirements. Now, for every function $f_{j} \in \mathfrak{F}$ and for every integer $k$, we choose a point $P_{j, k}$ such that $1-\frac{1}{k}<f_{j}\left(P_{j, k}\right)<1$ $+\frac{1}{k^{-}}$, when such a point exists. To verify that $K$ is separable, it is sufficient to show that $P_{j, k}(j, k=1,2, \ldots)$ is dense in $K$. For any point $P_{0}$ of $K$, by our above observation, there exists a continuous function $f(P)$ such that $0 \leqq f(P) \leqq 1$ in $K, f\left(P_{0}\right)=1, f(P)=0$ in the complement of a neighborhood $U$ of $P_{1}$ and at each point $P \neq P_{0}, f(P)<1$. By our assumption there exists a sequence $\left\{f_{j^{\prime}}(P)\right\}$ of $\tilde{j}$ such that $f(P)$ is a uniform convergence limit of $f_{j^{\prime}}(P)$. Let $\left\{P_{j^{\prime}}\right\}$ be a subsequence of $\left\{P_{j, k}\right\}$ such that

$$
1-\frac{1}{j^{\prime}}<f_{j^{\prime}}\left(P_{j^{\prime}}\right)<1+\frac{1}{j^{\prime}} .
$$

Then we see that $\lim _{\jmath^{\prime}} f_{j^{\prime}}\left(P_{j^{\prime}}\right)=1$. Let $\widetilde{P}$ be an accumulation point of $\left\{P_{j^{\prime}}\right\}$. Since $K$ satisfies the first axiom of countability, there exists a subsequence $\left\{P_{j^{\prime \prime}}\right\}$ of $\left\{P_{j^{\prime}}\right\}$ which tends to $\widetilde{P}$. It is easy to verify that $f(\widetilde{P})=\lim _{j^{\prime \prime}} f_{j^{\prime \prime}}\left(P_{j^{\prime \prime}}\right)=1$, and that $\widetilde{P}=P_{0}$, that is, $\left\{P_{j, k}\right\}$ is dense in $K$.

Finally, we shall prove that $K$ satisfies the second axiom of countability. For the purpose we shall show that, for any neighborhood $U$ of an arbitrary point $P_{0}$ of $K$, there exist a finite family $\left\{f_{l}\right\}\left(l=1,2, \ldots, m_{0}\right)$ of continuous functions of $\mathfrak{F}$, and a finite family $\left\{\varepsilon_{l}\right\}\left(l=1,2, \ldots, m_{0}\right)$ of positive numbers and a point $P_{n_{0}}$ belonging to the family $\left\{P_{j, k}\right\}$ chosen above, such that an open set

$$
N\left(P_{n_{v}} ; f_{l}, \varepsilon_{l}\right)=\left\{P ;\left|f_{l}(P)-f_{l}\left(P_{n_{0}}\right)\right|<\varepsilon_{l}, l=1,2, \ldots, m_{0}\right\}
$$

is a neighborhood of $P_{0}$ which is contained in $U$. In fact, there exist a finite family $\left\{f_{l}\right\}\left(l=1,2, \ldots, m_{0}\right)$ of continuous functions of $i \mathfrak{F}$ and a finite family $\left\{\varepsilon_{l}\right\}\left(l=1,2, \ldots, m_{0}\right)$ of positive numbers'such that an open neighborhood

$$
N\left(P_{0} ; f_{l}, 2 \varepsilon_{l}\right)=\left\{P ;\left|f_{l}(P)-f_{l}\left(P_{0}\right)\right|<2 \varepsilon_{l}, l=1,2, \ldots, m_{0}\right\}
$$


is contained in $U$. Since $\left\{P_{j, k}\right\}\{j, k=1,2, \ldots\}$ is dense in $K$, we can choose a point $P_{n_{0}}$ of $\left\{P_{j, k}\right\}$ such that

$$
\left|f_{l}\left(P_{n_{0}}\right)-f_{l}\left(P_{0}\right)\right|<\varepsilon \text { for } l=1,2, \ldots, m_{0} .
$$

We put

$$
N\left(P_{n_{0}} ; f_{l}, \varepsilon_{l}\right)=\left\{P ;\left|f_{l}(P)-f_{l}\left(P_{n_{0}}\right)\right|<\varepsilon_{l}, l=1,2, \ldots, m_{0}\right\},
$$

then we obtain that $P_{n} \in N\left(P_{n_{0}} ; f_{l}, \varepsilon_{l}\right)$ and $N\left(P_{n_{0}} ; f_{l}, \varepsilon_{l}\right) \subset N\left(P_{0} ; f_{l}, 2 \varepsilon_{l}\right) \subset U$. This shows that the family $\left\{N\left(P_{j, k} ; f_{l}, \varepsilon_{l}\right)\right\} \quad\left(f_{l} \in \tilde{f} ; l=1,2, \ldots, m ; j, k\right.$ $=1,2, \ldots$ ) of neighborhoods is a base of neighborhoods of an arbitrary point of $K$, and hence $K$ satisfies the second axiom of countability. Thus we conclude that $K$ is metrisable.

2. Now let $\mathscr{D}(P, Q)$ be a continuous real-valued function defined on the product space $\Omega \times \Omega$, which satisfies the following conditions:

$1^{\circ} 0<\phi(P, Q) \leqq+\infty$,

$2^{\circ} \Phi(P, Q)$ is finite except at most at the points of the diagonal set of $\Omega \times \Omega, 11$

$3^{\circ} \mathscr{D}(P, Q)$ is symmetric, that is, $\mathscr{D}(P, Q)=\mathscr{D}(Q, P)$. The potential $U^{\mu}(P)$ of a positive measure $\mu$ is defined by

$$
U^{\mu}(P)=\int \Phi(P, Q) d_{\mu}(Q)
$$

Then $U^{\mu}(P)$ is lower semi-continuous in $\Omega$ and continuous in $\Omega-S_{\mathrm{i}}$. By the condition $3^{\circ}$, we have always the reciprocal law, $\int U^{\mu} d \nu=\int U^{\nu} d \mu$, for any two positive measures $\mu$ and $\nu$. In this paper, $\mu$ will be called admissible on a compact set $K$, if $S_{\curlywedge} \subset K$ and $U^{\mu}(P) \leqq 1$ everywhere in $\Omega$. The family of all admissible measures on $K$ is denoted by $\mathfrak{?}(K)$. We associate every compact subset $K$ with the number $c(K)$ defined by sup $\mu(1)$ for all $\mu \in \Re(K)$. By this set-function $c(K)$ we define the inner and the outer capacities of an arbitrary subset $A$ of $\Omega$ as follows: the inner capacity $\operatorname{cap}_{i}(A)$ is equal to sup $c(K)$ for all compact sets $K \subset A$, and the outer capacity $\operatorname{cap}_{e}(A)$ is equal to $\inf \operatorname{cap}_{i}(G)$ for all open sets $G \supset A$. It follows immediately that we have $\operatorname{cap}_{i}(K)$ $=c(K)$ for every compact set $K, \operatorname{cap}_{i}(A) \leqq \operatorname{cap}_{e}(A)$ for an arbitrary subset

4) If $w(P, P)=+\infty$ at every point of the diagonal set, every compact subset of $Q$ is necessarily metrisable. 
$A$ and $\operatorname{cap}_{i}(G)=\operatorname{cap}_{e}(G)$ for every open set $G$. When the inner capacity $\operatorname{cap}_{i}(A)$ of $A$ is equal to the outer capacity $\operatorname{cap}_{e}(A)$, we shall say that $A$ is capacitable and we shall denote the common value of these two capacities by $\operatorname{cap}(A)$, which we shall call the capacity of $A$. Every open set is capacitable and as we shall show later, every compact $G_{\delta}$ set is capacitable. It may happen that an open set $G$ is of capacity zero; only in $\$ 4$ we assume that every open set is of positive capacity.

We say that a property holds nearly everywhere (resp. quasi everywhere) in a subset $A$, when the property holds at each point of $A$ except at the points of a set of inner (resp. outer) capacity zero.

3. The following theorems are well-known.

THEOREM 1.3. If $B_{n}(n=1,2, \ldots)$ are borelian sets, then we have

$$
\operatorname{cap}_{i}\left(\bigcup_{n=1}^{\infty} B_{n}\right) \leqq \sum_{n=1}^{\infty} \operatorname{cap}_{i}\left(B_{n}\right)
$$

THeOREM 1.4. For any sequence $\left\{A_{n}\right\}(n=1,2, \ldots)$ of arbitrary subsets of $\Omega$, it holds that

$$
\operatorname{cap}_{e}\left(\bigcup_{n=1}^{\infty} A_{n}\right) \leqq \sum_{n=1}^{\infty} \operatorname{cap} e\left(A_{n}\right)
$$

Theorem 1.5. If a sequence $\left\{\mu_{n}\right\}(n=1,2, \ldots)$ of positive measures converges vaguely to $\mu$, then we have

$$
U^{\mu}(P) \leqq \lim _{n} U^{\lambda_{n}}(P)
$$

at every point $P$ of $\Omega$.

4. Definition 1.1. We say that $\emptyset$ satisfies Frostman's maximum principle, if, for every potential $U^{\mu}$ such that $U^{\mu}(P) \leqq 1$ at every point $P$ of $S_{\mu}$, we have the same inequality everywhere in $\Omega$.

Definition 1.2. A positive measure $\mu_{K}$ assosiated with a compact set $K$ is called an equilibrium measure of $K$, if it holds the following properties:

$$
S_{\mu_{K}} \subset K, U^{\mu_{K}}(P) \leqq 1 \text { everywhere in } \Omega \text { and } U^{\mu_{K}}(P)=1 \text {. }
$$

nearly everywhere on $K$.

It is known that the equality, $\mu_{K}(1)=\operatorname{cap}_{i}(K)$, holds for an equilibrium measure $\iota_{K}$ of a compact set $K$. (See, for example, Theorem 3.5.) 
We call the potential $U^{\mu_{K}}$ of an equilibrium measure $\iota_{K}$ the equilibrium potential of $K$. For a compact set $K$ of inner capacity zero, we have $\mu_{K} \equiv 0$ as an equilibrium measure.

Definition 1.3. We shall say that a potential $U^{\mu}$ is quasi continuous in $\Omega$, if, for any $\varepsilon>0$, there exists an open set $G_{\text {: }}$ such that $\operatorname{cap}\left(G_{\varepsilon}\right) \leqq \varepsilon$ and the restriction of $U^{\mu}$ to $\Omega-G_{\varepsilon}$ is continuous.

Definition 1.4." We say that D satisfies the quasi continuity principle, if the continuity of the restriction of any potential $U^{\mu}$ to $S_{\mu}$ implies the quasi continuity of $U^{\mu}$ in $\Omega$.

Definition 1.5. We say that $D$ satisfies the continuity principle, if the continuity of the restriction of any potential $U^{\mu}$ to $S_{\mu}$ implies the continuity of $U^{\mu}$ in $\Omega$.

For the continuity principle, see Ohtsuka $[15,16,17]$, Kishi [10], Choquet [6] and Ninomiya [14]. The quasi continuity principle follows immediately from the continuity principle, but the latter does not follow from the former.

5. If every open set is of positive capacity, the existence of an equilibrium measure of every compact set $K$ implies Frostman's maximum principle. ${ }^{6)}$ and then it assures us the continuity principle." In our case, since there may exist an open set of capacity zero, we can only assert the following

THEOREM 1.6." Suppose that ue have an equilibrium measure of every compact set. Then $\Phi$ satisfies the quasi continuity principle.

To prove this theorem we shall use the following

THEOREM 1.7. There exists the largest open set $G_{0}$ of capacity zero, that is, $\operatorname{cap}\left(G_{0}\right)=0$ and, when $\Omega$ is of positive capacity, it holds that $\operatorname{cap}(G)>0$ for any open set $G=G_{0}$.

Proof. Let $\&$ be the family of all open sets of capacity zero. Then the open set $G_{0}=\bigcup_{G \in \mathbb{S}} G$ is the largest open set of capacity zero. In fact, for any compact set $K \subset G_{0}$, we have $\operatorname{cap}_{i}(K)=0$, since $K$ is covered by a finite number

5) Cf. Kishi [11].

6) Cf. Ninomiya [13].

7) See, for example, Ugaheri [19] or Ohtsuka [16].

8) We can construct a kernel function $\Phi$ in a suitable locally compact space $\Omega$ such that $\Phi$ does not satisfy the continuity principle, but, for any compact set $K$, there exists an equilibrium measure $\mu_{K}$. This example also shows that the continuity principle does not necessarily follow from the quasi continuity principle. 
of open sets of $\left(\xi\right.$, and hence $\operatorname{cap}\left(G_{0}\right)=0$. It follows immediately that, when $\Omega$.is of positive capacity, it holds that $\operatorname{cap}(G)>0$ for any open set $G \risingdotseq G_{0}$.

Now we shall give the proof of Theorem 1.6. Let the restriction of $U^{\mu}$ to $S_{\mu}$ be continuous. Without loss of generality, we may assume that $\left(\Omega-S_{\mu}\right)-G_{\text {v }}$ is not empty. It is sufficient to prove that the restriction of $U^{\mu}$ to $F_{0}=\Omega-G_{\text {n }}$ is continuous. Let $P_{0}$ be a point of $S_{\alpha} \cap F_{0}$. If $\Phi\left(P_{0}, P_{0}\right)$ is finite, $U^{i}(P)$ is obviously continuous at $P_{0}$, considered as a function in $Q$. Hence we suppose that $\Omega\left(P_{0}, P_{9}\right)=+\infty$. Let $\mu_{n}$ be the restriction of $\mu$ to $B_{n}=\left\{P ; \phi\left(P, P_{0}\right)>n\right\}$ $(n=1,2, \ldots)$. Then each potential $U^{\mu_{n}}$ is continuous on $S_{\mu}$ and it decreases uniformly to zero on $S_{\mu}$ as $n \rightarrow \infty$. We put $\varepsilon_{n}=\sup _{P \in s_{\mu}} U^{\mu_{n}}(P)$, then $\left\{\varepsilon_{n}\right\}$ decreases monotonously to zero. As $U^{\mu_{n}}(P) \leqq \varepsilon_{n}$ on $S_{\mu_{n}} \subset S_{\mu}$, it holds that $U^{\mu_{\mu}}(P) \leqq \varepsilon_{n}$ everywhere in $F_{0}$, since $G_{0}$ is the largest open set in $\Omega$.

Consequently we have

and hence

$$
\begin{aligned}
\lim _{r_{0} \ni P \rightarrow P_{0}} U^{\mu}(P) & \leqq \varlimsup_{F_{0} \ni P \rightarrow P_{0}} U^{\mu_{n}}(P)+\lim _{F_{0} \ni P \rightarrow P_{0}} U^{\mu-\mu_{n}}(P) \\
& \leqq \varepsilon_{n}+U^{\mu-\mu_{n}}\left(P_{0}\right) \leqq \varepsilon_{n}+U^{\mu}\left(P_{0}\right),
\end{aligned}
$$

$$
\varlimsup_{F_{0} \exists P \rightarrow P_{0}} U^{\mu}(P) \leqq U^{\mu}\left(P_{0}\right)
$$

This shows that the restriction of $U^{\mu}$ to $F_{0}$ is upper semi-continuous at $P_{0} \in F_{0} \cap S_{\mu}$.

\section{$\S 2$. Sequences of potentials}

1. In the sections 2.1 and 2.2 we assume the quasi continuity principle and we consider a sequence $\left\{U^{\mu_{\mu}}\right\}$ of potentials of positive measures $\mu_{2}$ converging vaguely to $\mu$.

First we shall prove

THEOREM 2.1.9) Every potential $U^{\mu}$ is quasi continuous in $\Omega$.

Proof. Since the set of points $P$ such that $U^{\mu}(P)=+\infty$ is a $G_{\delta}$ set of outer capacity zero, there is no loss of generality in assuming that $U^{\mu}$ is finite in $\Omega$. For any $\varepsilon>0$ and for any positive integer $n$, by Lusin's theorem, there exists a compact set $K_{n}$ such that $\mu\left(\Omega-K_{n}\right)<{ }_{2.4^{n}}^{\varepsilon}$ and $U^{\mu}$ is finite and continuous on $K_{n}$. Then the potential $U^{\mu_{n}}$ of the restriction $\mu_{n}$ of $\mu$ to $K_{n}$ is

9. For the Newtonian potentials this has been proved by Cartan [4], Proposition 5, 
continuous on $K_{n}$, and hence, by our quasi continuity principle, $U^{u_{n}}$ is quasi continuous in $\Omega$. Therefore, we have an open set $G_{n}$ such that the restriction $U^{\mu_{n}}$ to $Q-G_{n}$ is continuous and $\operatorname{cap}\left(G_{n}\right) \leqq \frac{\varepsilon}{2^{n+1}} \cdot$ Put

$$
B_{n}=\left\{P \in \Omega-G_{n} ; U^{\mu}(P)-U^{\mu_{n}}(P)>\frac{1}{2^{n}}\right\} .
$$

Then $B_{n}$ is open in $\Omega-G_{n}$ and $B_{n} \cup G_{n}$ is open in $\Omega$. Hence

$$
\operatorname{cap}\left(B_{n} \cup G_{n}\right) \leqq \operatorname{cap}_{i}\left(B_{n}\right)+\operatorname{cap}\left(G_{n}\right) \leqq \operatorname{cap}_{i}\left(B_{n}\right)+\frac{\varepsilon}{2^{n+1}} \cdot
$$

We shall show the inequality $\operatorname{cap}_{i}\left(B_{n}\right) \leqq \frac{\varepsilon}{2^{n+1}}$. For any compact subset $e \subset B_{n}$, let $\gamma$ be admissible on $e$. Then

$$
\frac{1}{2^{n}} \gamma(1)<\int\left(U^{\mu}-U^{\mu_{n}}\right) d \gamma=\int_{\Omega-K_{n}} U^{\gamma} d \mu \leqq \mu\left(\Omega-K_{n}\right)<\frac{\varepsilon}{2.4^{n}},
$$

whence we have $\gamma(1)<\frac{\varepsilon}{2^{n+1}}$ and $\operatorname{cap}_{i}(e) \leqq \frac{\varepsilon}{2^{n+1}}$. Thus we have seen that $\operatorname{cap}_{i}\left(B_{n}\right) \leqq \frac{\varepsilon}{2^{n+1}}$ and so $\operatorname{cap}\left(B_{n} \cup G_{n}\right) \leqq \frac{\varepsilon}{2^{n}}$. Hence we see that $\operatorname{cap}\left(G_{\varepsilon}\right)$ $\leqq \varepsilon$, where $G_{\varepsilon}=\cup_{n}\left(B_{n} \cup G_{n}\right)$. Then it follows that the restriction of $U^{\prime \prime}$ to $\Omega-G_{\S}$ is continuous, because it holds that $0 \leqq U^{\mu}(P)-U^{\mu_{n}}(P) \leqq \frac{1}{2^{n}}$ at every point $P$ of $\Omega-G_{\varepsilon}$ and $U^{\mu_{n}}(P)$ is continuous in $\Omega-G_{\dot{\imath}}$.

Theorem 2.2. ${ }^{10)}$ Suppose that $\mu_{n}(n=1,2, \ldots)$ are positive measures on a compact set such that $U^{\mu_{n}}(P) \leqq M<+\infty$ in $\Omega$ and that $\left\{\mu_{n}\right\}$ converges vaguely to $\mu$. Then we have, for any potential $U^{\nu} \leqq 1, \lim _{n} \int U^{\mu_{n}} d \nu=\int U^{\mu} d \nu$.

Proof. By Theorems 1.5 we have $U^{\mu}(P) \leqq \lim _{n} U^{\mu_{n}}(P)$ everywhere in $\Omega$. Hence we have

$$
\int U^{\mu} d \nu \leqq \int \lim U^{\mu_{n}} d \nu \leqq \lim \int U^{\mu_{n}} d \nu
$$

We shall show $\overline{\lim } \int U^{\mu_{n}} d \nu \leqq \int U^{\mathrm{i}} d \nu$. Since $U^{\nu}$ is quasi continuous in $\Omega$ by Theorem 2.1 , we can find, for any $\varepsilon>0$, an open set $G_{\varepsilon}$ such that $\operatorname{cap}\left(G_{\S}\right) \leqq \varepsilon$ and the restriction of $U^{\nu}$ to $Q-G_{\S}$ is continuous. Put

$$
f=\left\{\begin{array}{ccc}
U^{\prime} & \text { on } & \Omega-G_{\varepsilon} \\
0 & \text { in } & G_{\S} .
\end{array}\right.
$$

10) Cf. Brelot [3], Lemma 5. 
Then $f$ is upper semi-continuous. Hence we have a continuous function $g$ such that

$$
g \geqslant f \text { and } \int g d \mu \leqq \int f d \iota+\varepsilon=\int_{\Omega-6 ;} U^{2} d \mu+\varepsilon
$$

Then we see

$$
\begin{aligned}
\lim \int_{\alpha-G \varepsilon} U^{\nu} d \mu_{n} & =\lim \int f d \mu_{n} \leqq \lim \int g d \mu_{n}=\int g d \mu \\
& \leqq \int_{د-G \varepsilon} U^{\prime} d \mu+\varepsilon \leqq \int U^{2} d \mu+\varepsilon
\end{aligned}
$$

On the other hand it is easily seen that $\mu_{n}\left(G_{\varepsilon}\right) \leqq M \varepsilon$ and $\int_{G \varepsilon} U^{\nu} d \mu_{n} \leqq M \varepsilon$. In fact, for any compact set $e \subset G_{\varepsilon}$, the measure ${ }_{M}^{1} \mu_{n}^{\prime}$ is admissible on $e$, where $\mu_{n}^{\prime}$ is the restriction of $\mu_{n}$ to $e$. Hence

$$
\frac{1}{M^{\mu}} \mu_{n}^{\prime}(1) \leqq \operatorname{cap}\left(G_{\varepsilon}\right) \leqq \varepsilon .
$$

Therefore we have

$$
\widehat{\lim } \int U^{\prime} d \mu_{n} \leqq \int U^{\prime} d \mu+(M+1) \varepsilon
$$

Consequently we obtain

$$
\lim \int U^{\mu_{n}} d \nu=\varlimsup \lim \int U^{\nu} d \mu_{n} \leqq \int U^{\nu} d \mu=\int U^{\mu} d \nu
$$

2. The following theorem plays an important role in $\$ 3$.

THEOREM 2.3. ${ }^{11)}$ Let $\mu_{n}(n=1,2, \ldots)$ be measures on a compact set such that the potentials $U^{\mu_{n}}$ are uniformly bounded in $\Omega$. If $\left\{\mu_{n}\right\}$ converges vaguely to "l, we have

$$
\lim U^{\mu_{n}}=U^{\mu}
$$

guasi everywhere in $\Omega$.

Proof. By Theorem 1.5 we have $U^{\mu}(P) \leqq \lim U^{\mu_{n}}(P)$ everywhere in $\Omega$. Hence it is sufficient to prove that $U^{\mu}(P) \geq \lim U^{\mu_{n}}(P)$ quasi everywhere in $\Omega$. We put

$$
V_{n, m}(P)=\min \left(U^{\lambda_{n} n}(P), \ldots, U^{\lambda_{m}}(P)\right) \quad \text { for } m \geqslant n \text {, }
$$

11' C̣f. Kishi [11]. 
and

$$
V_{n}(P)=\inf \left(U^{\lambda_{u}}(P), U^{\lambda_{n-1}}(P), \ldots\right) .
$$

Then the sequence $\left\{V_{n, m}(P)\right\}(m=n, n+1, \ldots)$ decreases to $V_{n}(P)$ as $m \rightarrow \infty$ and the sequence $\left\{V_{n}(P)\right\}(n=1,2, \ldots)$ increases to $V(P)=\lim _{n} U^{\mu_{n}}(P)$ as $n \rightarrow \infty$. For any $\varepsilon^{\prime}>0$, we have an open set $G_{\xi^{\prime}}$ such that $\operatorname{cap}\left(G_{\xi^{\prime}}\right) \leqq \varepsilon^{\prime}$ and each $U^{\mu_{\mu}}(P)$ and $U^{\mu}(P)$ are continuous on $\Omega-G_{\varepsilon^{\prime}}$ by Theorem 2.1. For any positive number $\varepsilon$, we put

$$
E_{n, m}(\varepsilon)=\left\{P ; \quad V_{n, m}(P)-U^{\mu}(P)>\varepsilon\right\}
$$

and

$$
E_{n, m}^{\varepsilon^{\prime}}(\varepsilon)=\left\{P \in \Omega-G_{\varepsilon^{\prime}} ; V_{n, m}(P)-U^{\mu}(P)>\varepsilon\right\} .
$$

Then it is obvious that each $E_{n, m}^{\varepsilon^{\prime}}(\varepsilon)$ is open in $\Omega-G_{\Sigma^{\prime}}$ and $E_{n, m}^{\varepsilon^{\prime}}(\varepsilon) \cup G_{\Sigma^{\prime}}$ is open in 2 . Hence we easily obtain the inequalities

$$
\begin{aligned}
\operatorname{cap}_{e}\left(E_{n, m}(\varepsilon)\right) & \leqq \operatorname{cap}\left(E_{n, m}^{\varepsilon^{\prime}}(\varepsilon) \cup G_{\varepsilon^{\prime}}\right) \\
& \leqq \operatorname{cap}_{i}\left(E_{n, m}^{\varepsilon^{\prime}}(\varepsilon)\right)+\operatorname{cap}\left(G_{\xi^{\prime}}\right) \equiv \operatorname{cap}_{i}\left(E_{n, m}^{\Sigma^{\prime}}(\varepsilon)\right)+\varepsilon^{\prime} .
\end{aligned}
$$

We shall prove that $\lim _{n} \operatorname{cap}_{i}\left(E_{n, m}^{\xi^{\prime}}(\varepsilon)\right)=0$. We can see immediately that

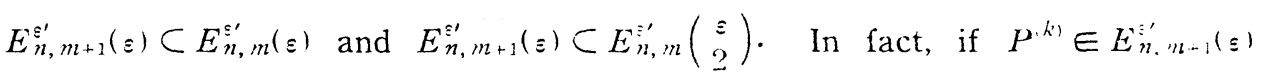
tends to $P_{0}$ as $k \rightarrow \infty$, then it follows that $P_{0} \in \Omega-G^{\prime}$ and that $\lim _{k} V_{n, m-1}\left(P^{\prime k^{\prime}}\right)$ $=V_{n, m+1}(P)$ and $\lim _{k} U^{\mu}\left(P^{(k)}\right)=U^{\prime}\left(P_{01}\right)$. If $\lim _{m} \operatorname{cap}_{i}\left(E_{n, m}^{\xi^{\prime}}(\varepsilon)\right)-\alpha>0$, we have, for any $m \geqslant n$, an admissible measure $r n, m$ on a compact subset $e_{n, m}$ of $E_{n, m}^{\varepsilon^{\prime}}(\xi)$

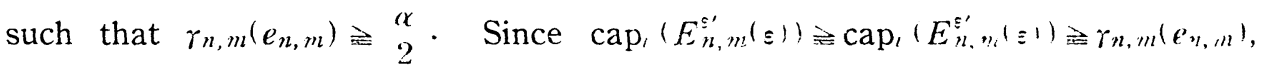
the total masses of $\gamma_{n, m}$ are uniformly bounded, and by Theorem 1.1, we can take out a subsequence $\left\{\gamma_{n, m^{\prime}}\right\}$ of $\left\{\gamma_{n, m}\right\}$ such that $\left\{\gamma_{n, m^{\prime}}\right\}$ converges vaguely to a positive measure $\gamma_{n}$, whose total mass is obviously not smaller than ${ }_{2}$. $S_{\gamma_{n}}$ is contained in $E_{n, m}^{\varepsilon^{\prime}}\left(\begin{array}{l}\varepsilon \\ 2\end{array}\right)$ for every sufficiently large $m$; otherwise there would be a point $P_{n} \in S_{i n}-E_{n, m_{n}}^{\varepsilon^{\prime}}\left(\begin{array}{c}\Sigma \\ 2\end{array}\right)$ for some $m_{n}$, and hence we could find a neighborhood $N$ of $P_{0}$ such that $N \cap E_{n, m_{0}-1}^{\varrho^{\prime}}(\varepsilon)=\psi$. Then $r_{n}(N)=0$ and $r n, m^{\prime}(N)$ $=0$ for every $m^{\prime} \geqslant m_{0}+1$, which is absurd. Since $S_{{ }_{n}} \subset E_{n, m}^{\varepsilon^{\prime}}\left(\begin{array}{l}\Xi \\ 2\end{array}\right)$. we have

$$
\alpha \varepsilon \leqq{ }_{2}^{\varepsilon} r_{n}(1) \triangleq j\left(V_{n, m}-U^{u}\right) d \tau_{n} \leqq j\left(U^{i_{m}}-U^{u}\right) d \gamma_{n}
$$

fore very sufficiently large $m$. On the other hand, we have $\lim _{m} \int U^{\mathrm{u}^{\prime} m} d \gamma_{n}=\int U^{\mu} d \gamma_{n}$ 
by Theorem 2.2. This contradicts (2). Consequently, we see that $\lim _{m} \operatorname{cap}_{i}\left(E_{n, m}^{\varepsilon^{\prime}}(\varepsilon)\right)=0$. Therefore, from (1), we can conclude that $\lim _{m}^{m} \operatorname{cap}_{e}\left(E_{n, m}(\varepsilon)\right) \leqq \varepsilon^{\prime}$. Thus we obtain $\lim _{m} \operatorname{cap}_{e}\left(E_{n, m}(\varepsilon)\right)=0$.

Now we choose a sequence $\left\{\varepsilon_{k}\right\}$ of positive numbers such that $\varepsilon_{1}>\varepsilon_{2}>\ldots$ $>\varepsilon_{k}>\varepsilon_{k+1}>\ldots \rightarrow 0$ and we put

$$
\begin{aligned}
& E_{n}\left(\varepsilon_{k}\right)=\left\{P ; \quad V_{n}(P)-U^{\mu}(P)>\varepsilon_{k}\right\} \\
& E\left(\varepsilon_{k}\right)=\left\{P ; \quad V(P)-U^{\mu}(P)>\varepsilon_{k}\right\}
\end{aligned}
$$

and

$$
E=\left\{P ; \quad V(P)-U^{\mu}(P)>0\right\} .
$$

Then, since it is immediately seen that $E_{n}\left(\varepsilon_{k}\right) \subset E_{n, m}\left(\varepsilon_{k}\right)$ and $E\left(\varepsilon_{k}\right)=\bigcup E_{n}\left(\varepsilon_{k}\right)$, we have $\operatorname{cap}_{e}\left(E_{n}\left(\varepsilon_{k}\right)\right)=0$ and $\operatorname{cap}_{e}\left(E\left(\varepsilon_{k}\right)\right)=0$. Then, from $E=\bigcup_{k} E\left(\varepsilon_{k}\right)$, we get $\operatorname{cap}_{e}(E)=0$, that is, $U^{\mu}(P) \geqq V(P)$ quasi everywhere in $\Omega$.

3. ThEOREM 2.4. Suppose that there exists an equilibrium measure of every compact set and that $\mu_{n}(n=1,2, \ldots)$ are measures on a compact set such that the potentials $U^{\mu_{n}} \leqq 1$ in $\Omega$. If $\left\{\mu_{n}\right\}$ converges vaguely to $\mu$, we have

$$
\frac{\lim }{n} U^{\mu_{n}}=U^{\mu}
$$

quasi everywhere in $\Omega$.

Proof. This follows immediately from Theorem 1.6 and 2.3.

4. When $\Phi$ satisfies the continuity principle, the uniform boundedness of $U^{\mu_{n}}(n=1,2, \ldots)$ is dispensable to assert that $U^{\mu}=\lim _{n} U^{\mu_{n}}$ quasi everywhere in $\Omega$.

For the purpose, first we shall show the following

Lemma 2.1. ${ }^{12}$ Let $\mu_{n}(n=1,2, \ldots)$ be measures on a compact set such that $\left\{\mu_{n}\right\}$ converges vaguely to $\mu$. Then, it holds that

$$
U^{\mu}=\underline{\lim } U^{\mu_{n}}
$$

nearly everywhere in $\Omega$.

Proof. We put

$$
E=\left\{P ; \quad V(P)-U^{\mu}(P)>0\right\}
$$

12) Cf. Brelot [3] and Ohtsuka [18]. 
where $V=\frac{\lim }{n} U^{i_{n}}$. If $\operatorname{cap}_{i}(E)>\alpha>0$, then we can find an admissible measure $\gamma$ on a compact set in $E$ such that $\gamma(1) \geqslant \alpha$. By Lusin's theorem, we have a restriction $\gamma^{\prime}$ of $r$ to a suitable compact subset of $S_{r}$ such that $\gamma^{\prime}(1) \geqslant \frac{\alpha}{2}$ and $U^{\gamma^{\prime}}$ is continuous on $S_{r^{\prime}}$. Then, by the continuity principle, $U^{\gamma^{\prime}}$ is continuous in $\Omega$. For this potential $U^{\gamma^{\prime}}$, we get

$$
0<\int\left(V-U^{\mu}\right) d r^{\prime} \leqq \lim \int U^{\mu_{n}} d r^{\prime}-\int U^{\mu} d r^{\prime}=0,
$$

which is impossible. Thus our lemma is established.

By our lemma, we have immediately

LEMMA 2.2. Let $\mu_{n}$ be measures on a compact set. If $\left\{\mu_{n}\right\}$ converges vaguely to $\mu$ and a potential $U^{r} \leqq 1$ in $\Omega$, then it holds that $\int U^{\mu} d r=\int V d r$, where $V=\frac{\lim }{n} U^{\mu_{n}}$.

THEOREM 2.5. ${ }^{13 i}$ Suppose that $\Phi$ satisfies the continuity principle. If $\mu_{n}$ $(n=1,2, \ldots)$ are measures on a compart set and $\left\{\mu_{n}\right\}$ converges vaguely to $h$, then we have

$$
U^{\mu}=\lim _{n} U^{\mu_{n}}
$$

quasi everywhere in $\Omega$.

Proof. We proceed in the same way as in the proof of Theorem 2.3. If $\lim _{m} \operatorname{cap}_{i}\left(E_{n, m}^{\varepsilon^{\prime}}(\varepsilon)\right)=\alpha>0$, then there exists an admissible measure $\gamma_{n}$, for which the inequality

$$
\frac{\alpha \varepsilon}{4} \leqq \int\left(V_{n, m}-U^{\mu}\right) d \gamma_{n}
$$

holds for every sufficiently large $m$. Here, letting $m$ tend to infinity, we have

$$
\frac{\alpha \varepsilon}{4} \leqq \int\left(V_{n}-U^{\mu}\right) d \gamma_{n} \leqq \int\left(V-U^{\mu}\right) d \gamma_{n} .
$$

The last integral of (3) is equal to zero by Lemma 2.2, which is absurd. Consequently, we have $\lim _{m} \operatorname{cap}_{i}\left(E_{n, m}^{\varepsilon^{\prime}}(\varepsilon)\right)=0$. Then, we can prove, by the same argument as in the proof of Theorem 2.3 , that $V=U^{\mu}$ quasi everywhere in $\Omega$.

13) See also Choquet [7]. 


\section{§3. Capacitability}

1. In this section we shall prove that every borelian and analytic set contained in a compact set are capacitable. We assume first that any compact set $K$ is metrisable. Then $K$ is a compact $G_{i}$ set and it can be concluded that any compact set $K$ is capacitable. We also assume that there exists an equilibrium measure $\mu_{K}$ of any compact set $K$. By our assumption we conclude that, for any open set $G$ contained in a compact set, there exists an equilibrium measure $\mu_{s_{i}}$ such that $\mu_{G}(1)=\operatorname{cap}(G), U^{\mu_{G}}(P) \leqq 1$ everywhere in $\Omega$ and $U^{\mu_{G}}(P)$ $=1$ quasi everywhere in $G$. By this fact we can prove that, if a sequence $\left\{X_{n}\right\}$ of arbitrary subsets contained in a fixed compact set $K$ increases monotously to $X$, then it holds that $\lim _{n} \operatorname{cap}_{e}\left(X_{n}\right)=\operatorname{cap}_{e}(X)$. Using Choquet's method, we can see that every borelian and analytic set contained in a compact set are capacitable.

2. We assume the following two conditions:

$1^{\circ}$ Any compact subset $K$ is metrisable.

$2^{\circ}$ There always exists an equilibrium measure $\mu_{K}$ of any compact subset $K$, that is, there exists a positive measure $\mu_{K}$ on $K$ such that $\mu_{K}(1)=\operatorname{cap}_{i}(K)$, $U^{\mu_{K}}(P) \leqq 1$ everywhere in $\Omega$ and $U^{\mu_{K}}(P)=1$ nearly everywhere on $K$.

THEOREM 3.1. If a decreasing sequence $K_{n}$ of compact subsets converges to $K$, then we have $\lim _{n} \operatorname{cap}_{i}\left(K_{n}\right)=\operatorname{cap}_{i}(K)$.

Proof. This theorem is proved without our two assumptions. For any $\varepsilon>0$ and for each $n$, there exists an admissible measure on $K_{n}$ such that $\operatorname{cap}_{i}\left(K_{n}\right)-\varepsilon \leqq \mu_{n}(1)$. As the total masses $\mu_{n}(1)$ of $\mu_{n}$ are uniformly bounded, we can take out a subsequence $\left\{\mu_{n^{\prime}}\right\}$ of $\left\{\mu_{n}\right\}$ which converges vaguely to $\mu_{0}$. We see that $S_{\mu_{0}}$ is contained in $K$. In fact, if there exists a point $P_{0} \in S_{\mu_{0}}-K$, then we can find a relatively compact neighborhood $\omega\left(P_{v}\right)$ such that $\overline{\omega\left(P_{0}\right)} \cap K$ $=\emptyset$. As $K$ is contained in $\Omega-\omega\left(P_{0}\right)$, there exists a sufficiently large $n_{0}$ such that, for all $n \geqslant n_{0}, \Omega-\overline{\omega\left(P_{0}\right)} \supset K_{n_{0}} \supset K$. Now let $f$ be a continuous function in $\Omega$ such that $0 \leqq f(P) \leqq 1, f(P)=1$ in $\omega\left(P_{0}\right)$ and $f(P)=0$ on $K_{n_{0}}$. Then we see that

$$
0<\int f d \mu_{0}=\lim _{n^{\prime}} \int f d \mu_{n}=0
$$

which is impossible. Hence $\mu_{0}$ is admissible on $K$ and $\mu_{0}(1) \triangleq \operatorname{cap}_{i}(K)$. Thus 
we have seen that $\lim _{n} \operatorname{cap}_{i}\left(K_{n}\right)-\varepsilon \leqq \operatorname{cap}_{i}(K)$, and hence $\lim _{n} \operatorname{cap}_{i}\left(K_{n}\right) \leqq \operatorname{cap}_{i}(K)$.

To prove the capacitability of compact set we prove the following

LеммA 3.1. If a compact set $K$ is a $G_{\delta}$ set, that is, $K=\bigcap_{n=1}^{\infty} G_{n}^{\prime}$, then there exists a sequence $\left\{G_{n}\right\}$ of open sets such that $G_{n}^{\prime} \supset G_{n}, G_{n} \supset G_{n+1} \supset G_{n+1} \ldots$, $G_{n}$ are compact and $K=\bigcap_{n=1}^{\infty} G_{n}$.

Proof. There is no loss of generality in assuming that each $G_{n}^{\prime}$ is relatively compact. For each $G_{n}^{\prime}$, there exists a continuous function $f_{n}$ in $\Omega$ such that $0 \leqq f_{n} \leqq 1$ in $\Omega, f_{n}(P)=0$ on $K$ and $f_{n}(P)=1$ on $\Omega-G_{n}^{\prime}$. We put

$$
f(P)=\sum_{n=1}^{\infty} \frac{1}{2^{n}} f_{n}(P)
$$

then $f$ is continuous in $\Omega$ and $0 \leqq f \leqq 1$ and $K=\{P ; f(P)=0\}$. The open sets $G_{n}=\left\{P ; f(P)<\frac{1}{2^{n-1}}\right\}(n=1,2, \ldots)$ satisfy the conditions required in our lemma. In fact, $K=\cap G_{n}$ and $\overline{G_{n}} \subset\left\{P ; f(P) \leqq \frac{1}{2^{n-1}}\right\}$ and hence $G_{n+1} \subset G_{n}$. In $\Omega-G_{n}^{\prime}$ it holds that $f_{m}(P)=1$ for all $m \geqslant n$ and hence $f(P) \geqslant \sum_{m=n}^{\infty} \frac{1}{2^{m}=} \begin{gathered}1 \\ 2^{n-1}\end{gathered}$. Consequently we have $G_{n}^{\prime} \supset G_{n}$.

\section{Lemma 3.2. Every compact $G_{\check{o}}$ set $K$ is capacitable.}

Proof. By Lemma 3.1, we can choose a sequence $\left\{G_{n}\right\}$ of open sets such that $G_{n} \supset \overline{G_{n+1}} \supset G_{n+1} \supset \ldots$, each $G_{n}$ is compact and $K=\cap G_{n}$. Then we have by Theorem 3.1 that

$$
\operatorname{cap}_{i}(K)=\lim \operatorname{cap}_{i}\left(\overline{G_{n}}\right) \geqslant \lim \operatorname{cap}_{i}\left(G_{n}\right) \geqslant \operatorname{cap}_{e}(K) .
$$

THEOREM 3.2. Every compact set is capacitable.

Proof. Since, by our assumption $1^{\circ}$, every compact set is a $G_{\delta}$ set, this follows immediately from Lemma 3.2.

THEOREM 3.3. An equilibrium potential $U^{\mu_{K}}$ of a compact set $K$ has the following properties: $\mu_{K}(1)=\operatorname{cap}(K), U^{\mu_{K}}(P) \leqq 1$ in $\Omega$ and $U^{\mu_{K}}(P)=1$ quasi everywhere on $K$.

Proof. It is sufficient to show that $U^{\mu_{K}}(P)=1$ quasi everywhere on $K$. We put

$$
E=\left\{P \in K ; U^{\mu_{K}}(P)<1\right\}
$$


and

$$
E_{n}=\left\{P \in K ; U^{\mu_{K}}(P) \leqq 1-\frac{1}{n}\right\} \quad(n=1,2, \ldots) .
$$

Then each $E_{n}$ is compact and $E=\cup E_{n}$. Since $\operatorname{cap}_{i}(E)=0$, we have $\operatorname{cap}_{i}\left(E_{n}\right)=0$ and hence, by Theorem 3.2 ,

$$
\operatorname{cap}_{e}(E) \leqq \sum_{n=1}^{\infty} \operatorname{cap}_{e}\left(E_{n}\right)=0
$$

The following theorem is very useful to estimate the outer capacity of a subset of $\Omega$.

THEOREM 3.4. Suppose that $U^{\mu}(P) \leqq 1$ in $\Omega$ and $U(P)=1$ quasi everywhere in $X$. Then we have $\operatorname{cap}_{e}(X) \leqq \mu(1)$.

Proof. Putting

and

$$
Y=\left\{P ; \quad U^{\mu}(P)=1\right\}
$$

$$
E=\left\{P \in X ; \quad U^{\mu}(P)<1\right\},
$$

we have $\operatorname{cap}_{e}(E)=0$ and $X=(X \cap Y) \cup E$, and hence

$$
\operatorname{cap}_{e}(X) \leqq \operatorname{cap}_{e}(X \cap Y)+\operatorname{cap}_{e}(E)=\operatorname{cap}_{e}(X \cup Y) \leqq \operatorname{cap}_{e}(Y) \text {. }
$$

Thus it is sufficient to show that $\operatorname{cap}_{e}(Y) \leqq \mu(1)$. We put

$$
Y_{n}=\left\{P ; U^{\mu}(P)>1-\frac{1}{n}\right\} \quad(n=2,3, \ldots),
$$

then each $Y_{n}$ is open and $\cap Y_{n}=Y$, hence $\lim _{n} \operatorname{cap}\left(Y_{n}\right) \geqq \operatorname{cap}_{e}(Y)$. For any $\varepsilon>0$ and each $n$, there exists an admissible measure $\mu_{n}$ on a compact subset of $Y_{n}$ such that $\operatorname{cap}\left(Y_{n}\right)-\varepsilon \leqq \mu_{n}(1)$. Here it follows that

$$
\left(1-\frac{1}{n}\right) \mu_{n}(1)<\int U^{\mu} d \mu_{n}=\int U^{\mu_{n}} d \mu \leqq \mu(1) .
$$

Consequently we have

$$
\operatorname{cap}_{i}\left(Y_{n}\right)-\varepsilon \leqq \frac{n}{n-1} \mu(1)
$$

and hence $\lim _{n} \operatorname{cap}\left(Y_{n}\right) \leqq \mu(1)$ and $\operatorname{cap}_{e}(Y) \leqq \mu(1)$.

Analogously to Theorem 3.4 , we have

THEOREM 3.5. Suppose that $U^{\mu}(P) \leqq 1$ in $\Omega$ and $U^{\mu}(P)=1$ nearly everywhere in $X$. Then the inequality $\operatorname{cap}_{i}(X) \leqq \mu(1)$ holds. 
THEOREM 3.6, For any relatively compact set $G$, there exists an equilibrium

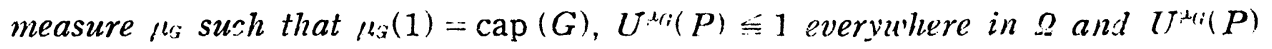
$=1$ quasi everywhere in $G$.

Proof. We can find an increasing sequence $\left\{K_{n}\right\}$ of compact sets such that $\cup K_{n}=G$. For each $K_{n}$, there exists an equilibrium potential $U^{\mu_{n}}$ which is, by Theorem 3.3, equal to 1 quasi everywhere on $K_{n}$. We can choose a subsequence $\left\{\ell_{n^{\prime}}\right\}$ of $\left\{\ell_{n}\right\}$ which converges vaguely to $\mu_{n}$, because $\mu_{n}(1) \leqq \operatorname{cap}(G)<+\infty$. It is seen that $\mu_{i}$ is an equilibrium measure of $G$. In fact, by Theorem 1.5, $U^{\mu_{0}}(P) \leqq \lim U^{\mu_{\mu^{\prime}}}(P) \leqq 1$ everywhere in $\Omega$ and $\mu_{j}(1)=\lim \mu_{n^{\prime}}(1)=\lim \operatorname{cap}\left(K_{n}\right)$ $\leqq$ cap $(G)$. We shall show that $U^{\mu_{n}}(P)=1$ quasi everywhere in $G$. Since for any $n^{\prime} \gg n_{3}^{\prime}, U^{\mu_{\mu^{\prime}}}(P)=1$ quasi everywhere on $K_{n_{0^{\prime}}}$, we have that $\lim U^{\mu_{i \prime^{\prime}}}(P)=1$ quasi everywhere on $K_{n_{v^{\prime}}}$, and hence, by Theorem 2.4, $U^{\mu_{0}}(P)=1$ quasi everywhere on $K_{n_{0^{\prime}}}$ and $U^{\mu_{0}}(P)=1$ quasi everywhere in $G$. Consequently, by Theorem 3.4 , we get $\operatorname{cap}(G) \leqq \mu_{1)}(1)$.

THEOREM 3.7. For an arbitrary subset $X$ contained in a compact set, there exists an equilibrium measure $\mu_{X}$ such that $\mu_{\mathrm{Y}}(1)=\operatorname{cap}_{e}(X), U^{\lambda_{1}}(P) \leqq 1$ every. where in $\Omega$ and $U^{\mu} X(P)=1$ quasi everywhere in $X$.

Proof. For a given $X$, there exists a sequence $\left\{G_{n}\right\}$ of open sets such that $G_{n} \supset X$ and $\operatorname{cap}_{e}(X)=\lim _{n} \operatorname{cap}\left(G_{n}\right)$, where we may suppose that each $G_{n}$ is relatively compact. For each $G_{n}$, there exists an equilibrium measure $\mu_{n}$ by Theorem 3.6. The total masses $\mu_{n}(1)$ being uniformly bounded, a subsequence $\left\{\mu_{n^{\prime}}\right\}$ of $\left\{\mu_{n}\right\}$ converges vaguely to $\mu_{0}$. Obviously it follows that $U^{\Lambda_{0}}(P) \leqq 1$ everywhere in $\Omega$ and, by Theorem 2. 4, we see that $U^{\mu_{0}}(P)=1$ quasi everywhere in $X$. We have also

$$
\mu_{0}(1)=\lim _{n^{\prime}} \mu_{n^{\prime}}(1)=\lim _{n} \operatorname{cap}\left(G_{n}\right)=\operatorname{cap}_{e}(X) .
$$

THEOREM 3.8. If an increasing seguence $\left\{X_{n}\right\}$ of arbitrary subsets converges to a relatively compact subset $X$, then we have $\lim _{c} \operatorname{cap}_{e}\left(X_{n}\right)=\operatorname{cap}_{e}(X)$.

Proof. By Theorem 3.7, there exists an equilibrium measure $\mu_{n}$ of each $X_{n}$. We can choose a subsequence $\left\{\mu_{n}\right\}$ of $\left\{\mu_{n}\right\}$ which converges vaguely to $\mu_{0}$. Obviously $U^{\mu_{0}}(P) \leqq 1$ everywhere in $\Omega$. Since, by Theorem $2.4, U^{\mu_{0}}(P)$ $=\lim U^{\mu_{h^{\prime}}}(P)$ quasi everywhere in $\Omega$, we see that $U^{\mu_{0}^{*}}(P)=1$ quasi everywhere in $X$. Hence, by Theorem 3.1, we obtain that 


$$
\operatorname{cap}_{e}(X) \leqq \mu_{0}(1)=\lim \mu_{n}(1)=\lim \operatorname{cap}_{e}\left(X_{n}\right) \leqq \operatorname{cap}_{e}(X) .
$$

3. ${ }^{14)}$ Now we consider a product space $E \times F$ of a compact subset $E$ of $\Omega$ and a compact auxiliary space $F$. We define a capacity $c(K)$ of a compact subset $K \subset E \times F$ by $c(K)=\operatorname{cap}$ (pr. $K$ ), where pr. $K$ means the canonical projection of $K$ on $E$. The definitions of the inner capacity $c_{i}(X)$ and the outer capacity $c_{e}(X)$ of an arbitrary subset $X \subset E \times F$ are evident.

THEOREM 3.9.

$1^{\circ}$ For any subset $X \subset E \times F$, $\operatorname{cap}_{i}($ pr. $X) \geqslant c_{i}(X)$.

$2^{\circ}$ For an open set $G \subset E \times F$, cap (pr. $\left.G\right)=c(G)$.

$3^{\circ}$ For any subset $X \subset E \times E$, $\operatorname{cap}_{e}(\operatorname{pr} . X)=c_{e}(X)$.

These assertions are easily verified. See also Benzécri [1].

Theorem 3.10. If a subset $X \subset E \times F$ is capacitable, then pr. $X$ is capacitable.

Proof. By Theorem 3.9, we have

$$
\operatorname{cap}_{i}(\text { pr. } X) \gtrsim c_{i}(X)=c_{e}(X)=\operatorname{cap}_{e}(\text { pr. } X) .
$$

Theorem 3.11. Every compact subset $K$ of $E \times F$ is capacitable.

Proof. This follows from the definition of $c(K)$ and Theorems 3.2 and 3.9.

THEOREM 3.12. If a decreasing sequence $\left\{K_{n}\right\}$ of compact subsets of $E \times F$ converges to $K$, then we have $\lim c\left(K_{n}\right)=c(K)$.

Proof. This follows immediately from the equalities $c\left(K_{n}\right)=\operatorname{cap}\left(\operatorname{pr} . K_{n}\right)$ and Theorem 3.1.

THEOREM 3.13. If an increasing sequence $\left\{X_{n}\right\}$ of subsets of $E \times F$ converges to $X$, then we have $\lim _{n} c_{e}\left(X_{n}\right)=c_{e}(X)$.

Proof. This is an immediate consequence of Theorems 3.8 and 3.9.

By Theorems 3.11, 12 and 13, we can prove

TheOREM 3.14. Every $K_{\text {øo }}$ set of $E \times F$ is capacitable.

This theorem can be proved in the same way as in [1].

4. TheOREM 3.15. Every K-borelian and more generally K-analytic set in a compact set of $\Omega$ are capacitable.

14) In this section 3.3 we apply Choquet-Benzecri's method [1] and [5]. 
Proof. This follows from Theorems 3.10 and 3.14 and the fact that every $K$-borelian subset and $K$-analytic subset in a compact set of a Hausdorff space $E$ are the canonical projection on $E$ of a $K_{s \delta}$ of the product space of $E$ and a compact auxiliary space. ${ }^{15}$

THEOREM 3.16. If a $K$-analytic set, contained in a $K_{\sigma}$ set, is of inner capacity zero, then it is of outer capacity zero.

Proof. This is an immediate consequence of Theorems 1.4 and 3.15.

\section{§. Function $m^{*}\left(P, U^{\wedge}\right)$}

1. In this section we assume that every open set is of positive capacity. We associate the function $m^{*}\left(P, U^{i}\right)$ with every potential $U^{\mu}$ of positive measure $\mu$ and consider the behavior of this function. We shall say that a constant $k$ is an E-lower bound of $U^{\mu}$ in an open set $\omega$, if a set of points $P \in \omega$ such that $U^{\mu}(P)<k$ is of inner capacity zero. It is easily seen that, for any open set $\omega$, there exists the maximum of $E$-lower bounds of $U^{\mu}$ in $\omega$, which we denote by $m^{*}\left(U^{\mu}, \omega\right)$. Then the function $m^{*}\left(P, U^{\mu}\right)$ is defined as follows :

$m^{*}\left(P, U^{\mu}\right)=\sup m^{*}\left(U^{\mu}, \omega(P)\right)$ for all open neighborhoods of $P$.

It is well-known ${ }^{16)}$ that $m^{*}\left(P, U^{\mu}\right)$ is lower semi-continuous in $\Omega$ and $U^{\mu}(P)$ $\leqq m^{*}\left(P, U^{\mu}\right)$ everywhere in $\Omega$, as $U^{\mu}$ is lower semi-continuous. When $m^{*}\left(P, U^{\mu}\right)$ is continuous in an open set $\omega, U^{\mu}$ is called E-continuous in $\omega$.

THEOREM 4.1. If $U^{\mu}$ is E-continuous in $\omega$, then the equality $m^{*}\left(P, U^{\mu}\right)$ $=M\left(P, U^{i l}\right)$ holds everywhere in $\omega$, where $M\left(P, U^{\mu}\right)$ means the upper limit function of $U^{i}$.

Proof. We put $\varphi(P)=m^{*}\left(P, U^{\mu}\right)$, then $\varphi(P)$ is continuous in $\omega$, and hence $\varphi(P)=M(P, \varphi)$ everywhere in $\omega$. Since it holds that $\varphi(P) \triangleq U^{\mu}(P)$ everywhere in $\omega$, we have

$$
\varphi(P)=M(P, \varphi) \gtrsim M\left(P, U^{\mu}\right) \gtrsim m^{*}\left(P, U^{\mu}\right)=\varphi(P)
$$

everywhere in $\omega$. Consequently we get $m^{*}\left(P, U^{\mu}\right)=M\left(P, U^{\mu}\right)$.

THEOREM 4.2. Suppose that there exists a continuous function $\psi$ in an open

15) Cf. Choquet [5].

16. Cf. Hahn [9]. 
set w) which differs from $U^{4}$ at most at a set of points of inner capacity zero. Then $U^{\lambda}$ is E-continuous in $\omega$.

Proof. Let $P_{0}$ be a point of $\omega$. We shall show that, for every neighborhood $\omega\left(P_{0}\right) \subset \omega$, the equality $m^{*}\left(U^{\mu}, \omega\left(P_{0}\right)\right)=\inf _{\Gamma \in \omega\left(P_{0}\right)} \psi(P)$ holds. Put $k=m^{*}\left(U^{i L}, \omega\left(P_{0}\right)\right)$, then the set of points $P \in \omega\left(P_{0}\right)$ such that $U^{\mu}(P)<k$ is of inner capacity zero, hence $\psi(P) \geqslant k$ at every point $P$ of $\omega\left(P_{0}\right)$, because every open set is of positive capacity. Therefore, we obtain $\inf _{P \in \omega\left(P_{0}\right)} \psi(P) \geqq m^{*}\left(U^{\mu}, \omega\left(P_{0}\right)\right)$.

Now let $k$ be a constant which is smaller than $\inf _{P \subseteq \omega\left(P_{0}\right)} \phi(P)$. Then, since $\psi=U^{\mu}$ nearly everywhere in $\omega\left(P_{0}\right), k$ is an $E$-lower bound, and we get $k \leqq m^{*}\left(U^{\mu}, \omega\left(P_{0}\right)\right)$. This shows that $\inf _{P \in \omega\left(P_{0}\right)} \psi(P) \leqq m^{*}\left(U^{\mu}, \omega\left(P_{0}\right)\right)$. Consequently we have that $m^{*}\left(P_{0}, U^{\mu}\right)=\psi\left(P_{0}\right)$ at every point $P_{0} \in \omega$, and that $U^{\mu}$ is $E$ continuous in $\omega$.

Conversely we can prove

THEOREM 4.3. If $U^{\mu}$ is E-continuous in $\Omega$, then there exists a continuous function $\psi$ in $\Omega$ which coincides with $U^{\mu}$ nearly everywhere in $\Omega$.

To prove this theorem, we shall prove

THEOREM 4.4. If $U^{\mu}$ is E-continuous in $\Omega$, then $m^{*}\left(P, U^{\mu}\right)=U^{\mu}(P)$ nearly everywhere in $\Omega$.

Proof. We put

$$
E_{n}=\left\{P ; m^{*}\left(P, U^{\mu}\right)>U^{\mu}(P)+\frac{1}{n}\right\} \quad(n=1,2, \ldots)
$$

and

$$
E_{\infty}=\left\{P ; m^{*}\left(P, U^{\mu}\right)>U^{\mu}(P)\right\} .
$$

Then $E_{\infty}=\cup E_{n}$. It is sufficient to show that cap $c_{i}\left(E_{n}\right)=0$. We can easily see that $E_{n} \subset S_{\mu}$. For each $P_{0} \in S_{\mu}$, we can take a neighborhood $\omega_{n}^{\prime}\left(P_{0}\right)$ such that

$$
m^{*}\left(P, U^{\mu}\right)+\frac{1}{2 n}>m^{*}\left(P, U^{\mu}\right) \text { at every point } P \in \omega_{n}^{\prime}\left(P_{0}\right)
$$

and a neighborhood $\omega_{n}^{\prime \prime}\left(P_{0}\right)$ such that

$$
m^{*}\left(U^{\mu}, \omega_{n}^{\prime \prime}\left(P_{0}\right)\right)+\frac{1}{2 n}>m^{*}\left(P_{0}, U^{\mu}\right)
$$

hence we have 


$$
\begin{aligned}
& m\left(U^{\mu} \cdot \omega_{n}\left(P_{0}\right)\right)+\frac{1}{n}>m\left(P, U^{\mu}\right) \text { at every point } \\
& \qquad P \in \omega_{n}^{\prime \prime}\left(P_{0}\right)=\omega_{n}^{\prime}\left(P_{0}\right) \cap \omega_{n}^{\prime \prime}\left(P_{0}\right) .
\end{aligned}
$$

As $m^{*}\left(U^{\mu}, \omega_{n}\left(P_{1}\right)\right)$ is an E-lower bound of $U^{2 \mu}$ in $\left(_{n}\left(P_{1}\right)\right.$, we get $U^{\mu}\left(P^{\prime}\right)$ $\geq m^{*}\left(U^{\mu}, \omega_{n}\left(P_{1}\right)\right)$ nearly everywhere in $\omega_{n}\left(P_{11}\right)$. Consequently, $U^{\mu}(P)+\begin{aligned} & 1 \\ & n\end{aligned}$ $m\left(P, U^{\mu}\right)$ nearly everywhere in $\left({ }^{\prime}\right)_{n}\left(P_{n}\right)$, and hence $\operatorname{cap}_{i}\left(E_{n} \cap \omega_{n}\left(P_{0}\right)\right)=0$. Since $S_{\iota^{\alpha}}$ is compact, we have $\operatorname{cap}_{i}\left(E_{n}\right)=0$ and $\operatorname{cap}_{i}\left(E_{\infty}\right)=0$, that is, $m^{*}\left(P, U^{\wedge}\right)$ $\leqq U^{\mu}(P)$ nearly everywhere in $\Omega$.

Here we shall give the proof of Theorem 4.3. ${ }^{11}$

By Theorem 4.4, we can take a continuous function $\psi(P)=m\left(P, U^{u}\right)$, which coincides with $U^{\mu}$ nearly everywhere in 2 .

We shall say that $\Phi$ is equal to zero at infinity, if, for any fixed point $P \in \Omega, \mathscr{D}(P, Q)$ tends to zero as $Q$ tends to $\omega^{*}$, where $\omega^{*}$ is the Alexandroff's point of $\Omega$.

If a point $P_{0} \in S_{1}$ has such a property that, for any neighborhood $\omega\left(P_{0}\right)$ of $\left.P_{0}, \operatorname{cap}_{\imath}(\omega)\left(P_{0}\right) \cap S_{\mu}\right)>0$, then we say that $P_{0}$ belongs to $\widetilde{S}_{\mu}$.

THeOREM 4.5. Suppose that $\Phi$ is equal to zero at infinity, and the restriction of $U^{\mu}$ to $S_{\Lambda}$ is continuous at $P_{0} \in S_{\Lambda}$. If $U^{\Lambda}$ coincides with a continuous function $\dot{\psi}$ nearly everywhere in a neighborhood $\omega\left(P_{0}\right)$ of $P_{0}$, then $U^{\mu}$ is continuous at $P_{0}$.

Proof. Since $U^{\mu}$ coincides with $\psi$ nearly everywhere in $\omega\left(P_{0}\right)$, the function $m^{*}\left(P, U^{\mu}\right)$ is continuous in $\omega\left(P_{0}\right)$ by Theorem 4.2 , and $U^{*}$ is bounded in a neighborhood $\omega^{\prime}\left(P_{v}\right)$ of $P_{0}$ such that $\omega^{\prime}\left(P_{0}\right) \subset \omega^{\prime}\left(P_{v}\right) \subset \omega\left(P_{0}\right)$. We assert that $P_{0}$ belongs to $\widetilde{S}_{\mu}$. In fact, if $P_{0} \in S_{\mu}-\widetilde{S}_{\mu}$, then we have a neighborhood $\omega^{\prime \prime}\left(P_{0}\right)$ $\subset \omega^{\prime \prime}\left(P_{v}\right) \subset \omega^{\prime}\left(P_{v}\right)$ such that $\operatorname{cap}_{i}\left(\omega^{\prime \prime}\left(P_{0}\right) \cap S_{L}\right)=0$. On the other hand, the potential $U^{\mu^{\prime}}$ of the restriction $\mu^{\prime}$ of $\mu$ to $\overline{\omega^{\prime \prime}\left(P_{0}\right)}$ is bounded in $Q$, because $U^{\mu^{\prime}}$ $\leqq U^{\mu}$ and $U^{i}$ is bounded in $\omega^{\prime}\left(P_{0}\right)$ and $D$ is equal to zero at infinity. Consequently, we have $\iota^{\prime} \equiv 0$, which contradicts the assumption that $P_{v} \in S_{\mu}$.

Now, for any $\varepsilon>0$, there exist two neighborhoods $\omega_{1}\left(P_{0}\right)$ and $\omega_{2}\left(P_{0}\right)$ of $P_{0}$ such that

and

$$
m^{*}\left(P_{0}, U^{2}\right)-\varepsilon \leqq m^{*}\left(U^{\mu} \cdot \omega_{1}\left(P_{0}\right)\right)
$$

$$
U^{\mu}(P)<U^{\prime}\left(P_{1}\right)+\equiv \text { at every point } P_{i} \text { of } \quad \omega_{:}(P) \cap S_{i} .
$$

17. When $S$, is separable, this has been proved in Hahn [9], p. 175 . 
Then, in $\pi\left(P_{0}\right) \cap S_{1}$, where $\pi\left(P_{0}\right)=()_{1}\left(P_{0}\right) \cap \omega_{2}\left(P_{0}\right)$, we have

$$
m^{*}\left(P_{0}, U^{\mu}\right)-\Xi \leqq m^{*}\left(U^{\mu}, \widetilde{\omega}\left(P_{0}\right)\right)
$$

and

$$
U^{\mu}(P)<U^{\mu \cdot}\left(P_{0}\right)+\varepsilon .
$$

It follows that $m^{*}\left(U^{\mu}, \widetilde{\omega}\left(P_{0}\right)\right) \leqq U^{\mu}\left(P_{0}\right)+\varepsilon$. In fact, if $m^{*}\left(U^{\mu}, \widetilde{\omega}\left(P_{0}\right)\right)>U^{\mu}\left(P_{0}\right)$ $+\varepsilon$, then $S_{\mu} \cap \widetilde{\omega}\left(P_{0}\right)$ is contained in the set $\left\{P \in \hat{\omega}\left(P_{0}\right) ; U^{\mu}(P)<m^{*}\left(U^{\mu}, \widetilde{\omega}\left(P_{0}\right)\right)\right\}$, which contradicts the fact $P_{0} \in \widetilde{S}_{\mu}$. Thus we have seen that

$$
m^{*}\left(P_{0}, U^{\mu}\right)-\varepsilon \leqq m^{*}\left(U^{\mu}, \widetilde{\omega}\left(P_{0}\right)\right) \leqq U^{\mu}\left(P_{0}\right)+\varepsilon,
$$

and hence $m^{*}\left(P_{0}, U^{\mu}\right)=U^{\mu}\left(P_{0}\right)$.

Since $m^{*}\left(P_{1}, U^{\mu}\right)$ is continuous at $P_{0}$, there exists a neighborhood $\omega^{\prime}\left(P_{0}\right)$ of $P_{0}$ such that

$$
m\left(P_{0}, U^{\mu}\right)+\varepsilon>m^{*}\left(P, U^{\mu}\right) \text { at every point } P \in \omega^{\prime}\left(P_{0}\right) .
$$

Hence $U^{\mu}\left(P_{0}\right)+\varepsilon>m^{*}\left(P, U^{\mu}\right) \geqq U^{\mu}(P)$ at every point $P \in \omega^{\prime}\left(P_{0}\right)$. This means that $U^{\mu}(P)$ is upper semi-continuous at $P_{0}$.

Corollary. Suppose that $\Phi$ is equal to zero at infinity, and the restriction of $U^{\mu}$ to $S_{\alpha}$ is continuous. If there exists a continuous function $\psi$ in $\Omega$ such that $\varphi=U^{\mu}$ nearly everywhere in $\Omega$, then $U^{\mu}$ is continuous in $\Omega$.

2. Now we consider a potential $U^{\mu}(P)$, which coincides with $m^{*}\left(P, U^{\mu}\right)$ everywhere in $\Omega$.

TheOREM 4.6. A potential $U^{\mu}(P)$ coincides with $m^{*}\left(P, U^{\mu}\right)$ everywhere in $\Omega$ if and only if it has the following property: at every point $P_{0} \in \Omega$,

$$
\operatorname{cap}_{i}\left(\left\{P \in \omega\left(P_{0}\right) ; U^{\mu}(P) \leqq h\right\}\right)>0
$$

for any neighborhood $\omega\left(P_{0}\right)$ and $h>U^{\mu}\left(P_{0}\right) \cdot{ }^{1 s}$

Proof. Suppose that $U^{\mu}\left(P_{0}\right)<m^{*}\left(P_{0}, U^{\mu}\right)$, then there exist a neighborhood ${ }_{\omega}\left(P_{n}\right)$ of $P_{0}$ and a positive number $\varepsilon$ such that

$$
U^{\mu}\left(P_{0}\right)<m^{*}\left(U^{\mu}, \omega\left(P_{0}\right)\right)-\varepsilon \leqq m^{*}\left(U^{\mu}, \omega\left(P_{0}\right)\right) \leqq m^{*}\left(P_{0}, U^{\mu}\right) .
$$

Then the set $\left\{P \in \omega\left(P_{0}\right) ; U^{\mu}(P) \leqq n^{*}\left(U^{\mu}, \omega\left(P_{0}\right)\right)-\varepsilon\right\}$ is of inner capacity zero.

18. Choquet [7] assumes this condition to state the result that if $U^{\mu \cdot 1}=U^{\mu^{2}}$. nearly everywhere in 0 , we have $U^{\prime \prime}=U^{N_{2}}$ everywhere in $S$. 
Now suppose that $U^{\mu}\left(P_{0}\right)=m^{*}\left(P_{0}, U^{\mu}\right)$. Then, for any neighborhood $\omega\left(P_{0}\right)$ of $P_{0}$, we have $m^{*}\left(U^{\mu}, \omega\left(P_{0}\right)\right) \leqq U^{\mu}\left(P_{0}\right)$. Therefore, for any $h>U^{\mu}\left(P_{0}\right)$, the set $\left\{P \in \omega\left(P_{0}\right) ; U^{\mu}(P) \leqq h\right\}$ must be of inner capacity positive.

Hereafter, we assume that every compact set is separable.

THEOREм 4.7. Suppose that there exists an equilibrium measure of every compact set. If, for any potential $U^{\mu}, U^{\mu}(P)=m^{*}\left(P, U^{\mu}\right)$ everywhere in $\Omega$, then there exists an equilibrium potential $U^{\mu_{G}}$ for any open set contained in a compact set such that $\mu_{G}(1)=\operatorname{cap}(G), U^{\mu_{f_{F}}}(P) \leqq 1$ everywhere in $\Omega$ and $U^{\mu_{G}}(P)=1$ every. where in $G$.

Proof. Let $G$ be an open set contained in a compact set. There exists an increasing sequence $\left\{K_{n}\right\}$ of compact sets such that $K_{n} \subset G$ and $\cup K_{n}=G$. Let $\mu_{n}$ be an equilibrium measure of $K_{n}$, then a subsequence $\left\{\mu_{n^{\prime}}\right\}$ of $\left\{\mu_{n}\right\}$ converges vaguely to $\mu_{0}$. It is easily seen by Theorems $1.5,2.4$ and 3.5 that $U^{\mu_{0}} \leqq 1$ everywhere in $\Omega, U^{\mu_{0}}=1$ nearly everywhere in $G$ and $\mu_{0}(1)=\operatorname{cap}(G)$. Suppose that there exists a point $P_{0} \in G$ such that $U^{\mu_{0}}\left(P_{0}\right)<1$. Then there exist a neighborhood $\omega\left(P_{0}\right) \subset G$ and a positive number $\delta$ such that

$$
U^{\mu_{0}}\left(P_{n}\right)=m^{*}\left(P_{0}, U^{\mu_{0}}\right)<m^{*}\left(U^{\mu_{0}}, \omega_{1}\left(P_{0}\right)\right)+\delta<1 .
$$

Hence the set

$$
\left\{P \in \omega\left(P_{0}\right) ; \quad U^{\mu_{0}}(P) \leqq m^{*}\left(U^{\mu_{0}}, \omega\left(P_{0}\right)\right)+\delta\right\}
$$

is of inner capacity positive by Theorem 4.7, which contradicts the fact that $U^{\mu_{0}}(P)=1$ nearly everywhere in $G$. Thus we have seen that $U^{\mu_{0}}(P)=1$ everywhere in $G$.

TheOREM 4.8. Suppose that $\Phi$ satisfies Cartan's maximum principle ${ }^{191}$ and that there exists a potential $U^{\mu_{G}}$, for any open set $G$, such that $U^{\mu_{G}}(P)=1$ everywhere in $G$, and $U^{\mu_{G}}(P)=1$ nearly everywhere on $S_{\mu_{i}} \subset \bar{G}$. Then, for any potential $U^{\mu}$, we have $U^{\mu}(P)=m^{*}\left(P, U^{\mu}\right)$ everywhere in $\Omega$.

Proof. Suppose that there exist a constant $h$ and a neighborhood $\omega\left(P_{0}\right)$ of $P_{0}$ such that $U^{\mu}\left(P_{0}\right)<h$ and the set

$$
\left\{P \in \bar{\omega}\left(P_{0}\right) ; \quad U^{\mu}(P)<h\right\}
$$

19) Cartan's maximum principle means: Let $\mu$ be a positive measure with finite energy and ${ }^{\prime \prime}$ be an arbitrary positive measure. If $U^{\prime \prime} \leqq U^{*}$ nearly everywhere on $S_{i}$, then $U^{i} \leqq U^{2}$ everywhere in $[$. 
is of inner capacity zero. Let $U^{\text {a., }}$ be a potential assured by our assumption such that $U^{u_{\omega \prime}}(P)=1$ everywhere in $\omega\left(P_{0}\right)$ and $U^{\mu_{w}}(P)=1$ nearly everywhere on $S_{\mu, v} \subset \omega\left(P_{0}\right)$. Then it holds that $U^{\mu}(P) \geqslant h U^{\mu \cdot \omega}(P)$ nearly everywhere on (1) $\left(P_{0}\right) \supset S_{s_{\infty}}$ and hence, by Cartan's maximum principle, we see that $U^{: L}(P)$ $\geqq h U^{\mu_{10}(P)}$ everywhere in $\Omega$ and $U^{\mu}\left(P_{0}\right) \geqq h U^{\mu_{10}}\left(P_{0}\right)=h$, which is a contradiction.

3. Now we shall state an application of Theorem 4.7.

We shall say that a set $E$ is thin at a point $P_{11}$ if there exists a positive measure $y$ such that

$$
\lim _{E \ni \Gamma \rightarrow P_{0}} U^{2}(P)>U^{2}\left(P_{0}\right)
$$

THEOREM 4.9. Suppose that $E$ is of outer capacity zero. Then $E$ is thin at each point $P_{n} \in E$, where $\emptyset\left(P_{n}, P_{0}\right)=+\infty$.

Proof. Let $P_{0} \in E$ and $\Phi\left(P_{0}, P_{0}\right)=+\infty$. Put

$$
B_{n}=\left\{P ; \emptyset\left(P_{0}, P\right)>n\right\} \quad(n=1,2, \ldots) .
$$

Then $E_{n}=\left(B_{n-1}-B_{n}\right) \cap E\left(B_{0}=\Omega\right)$ is of outer capacity zero and there exists an open set $G_{n}$ such that $E_{n} \subset G_{n}, G_{n} \subset B_{n-1}-B_{n+1}$ and cap $\left(G_{n}\right)<\frac{1}{n(n+1) 2^{n}}$. Let $\mu_{n}$ be an equilibrium measure of $G_{n}$ and $\nu_{n}=n \mu_{n}$. Then $\nu_{n}(1)<\frac{1}{(n+1) 2^{n}}$ and $U^{\nu_{n}}(P)=n$ everywhere in $G_{n}$ by Theorem 4.8. We put $\nu=\sum \nu_{n}$. At a point $P \in\left(B_{n-1}-B_{n+1}\right) \cap E$, we have $U^{\nu}(P) \geqslant U^{\prime \prime \prime}(P)=n$ and $U^{\nu}\left(P_{0}\right)$ $=\sum U^{\nu_{n}}(P)<\sum(n+1) \nu_{n}(1)=1$, and hence

$$
\lim _{r \ni P \rightarrow l_{1,}^{\prime}} U^{\prime \prime}(P)>U^{\prime}\left(P_{n}\right)
$$

This shows that $E$ is thin at $P_{0}$.

Added in proofs: During the proofs of this paper, the author finds that Theorem 1.2 is established by Z. Semadeni and P. Zbijewski : Spaces of continuous functions (I), Studia Math, 16 (1957), 130-141.

\section{RefERENCES}

[1] J. P. Benzécri: Théorie des capacités, Seminaire Bourbaki, (1955).

[2] N. Bourbaki: Intégration, Paris, 1952.

[3] M. Brelot: Nouvelle démonstration du théorème fondamental sur la convergence des potentiels, Ann. Inst. Fourier, 6 (1955-1956), 361-368. 
[4] H. Cartan: Théorie du potentiel newtonien, énergie, capacité, suites de potentiels, Bull. Soc. Math., 73 (1945), 74-106.

[ 5 ] G. Choquet, Theory of capacities, Ann. Inst. Fourier, 5 (1953-1954), 131-295.

[6] G. Choquet: Les noyaux réguliers en théorie du potentiels, C. R. Acad. Sci., Paris, 243 (1956), 635-638.

[7] G. Choquet: Sur les fondements de la théorie fine du potentiel, ibid., 244 (1957), 1606-1609.

[8] O. Frostman; Potentiel d'équilibre et capacité des ensembles avec quelques applications à la théorie des fonctions, Thèse, Lund, (1935), 1-118.

[ 9 ] H. Hahn: Theorie der reellen Funktionen, Bd. 1, Berlin, 1921.

[10] M. Kishi: On a theorem of Ugaheri, Proc. Japa. Acad., 32 (1956), 314-319.

[11] M. Kishi : Inferior limit of a sequence of potentials, ibid., 33 (1957), 314-319.

[12] N. Kryloff and N. Bogoliouboff : La théorie general de la mesure dans son application à l'étude des systemes dynamiques de la mécanique non linéaire, Ann. of Math.. 38 (1937), 65-113.

[13] N. Ninomiya: Sur le théorème du balayage et le théorème de d'équilibre, Jour. Inst. Polytech. Osaka City Univ., 6 (1955), 83-91.

[14] N. Ninomiya: Sur le principe de continuité dans la théorie du potentiel, ibid., 8 (1957), 51-56.

[15] M. Ohtsuka: Sur un espace complet de mesures positives dans la théorie du potentiel, Proc. Japan Acad., 32, (1956), 311-313.

[16] M. Ohtsuka: Sur un théorème de M. Kishi, ibid., 32 (1956), 722-725.

[17] M. Ohtsuka: Les relations entre certains principes en théorie du potentiel, ibid., 33 (1957), 37-40.

[18] M. Ohtsuka: Selected topics in the theory of functions, Tokyo, 1957 (in Japanese).

[19] T. Ugaheri: On the general capacities and potentials, Bull. Tokyo Inst. Tech., 4 (1953), 149-179.

\section{Mathematical Institute}

Nagoya University 